\title{
The Phylogenomics of CRISPR-Cas System in Salmonella: an evolutionary race with
}

\section{housekeeping genes}

Simran Krishnakant Kushwaha ${ }^{*}$, Chandrajit Lahiri ${ }^{2}$, Bahaa Abdella ${ }^{2,3}$, and Sandhya Amol

$$
\text { Marathe }^{1 *}
$$

${ }^{1}$ Department of Biological Sciences, Birla Institute of Technology \& Science (BITS), Pilani, Rajasthan, India

${ }^{2}$ Department of Biological Sciences, Sunway University, Petaling Jaya, Selangor, Malaysia ${ }^{3}$ Faculty of Aquatic and Fisheries Sciences, Kafrelsheikh University, Kafrelsheikh, Egypt

\section{*Corresponding authors:}

Sandhya Amol Marathe. E-mail: sandhya.marathe@pilani.bits-pilani.ac.in

Simran Krishnakant Kushwaha. E-mail: p20180406@pilani.bits-pilani.ac.in 


\begin{abstract}
Salmonellae display intricate evolutionary patterns comprising over 2500 serovars having diverse pathogenic profiles. The acquisition/exchange of various virulence factors influence the evolutionary framework. To gain insights into evolution of Salmonella as a pathogen in association with the CRISPR-Cas genes we performed phylogenetic surveillance across strains of 22 Salmonella serovars. The strains assorted into two main clades, pertaining to the differences in their CRISPR1-leader and cas operon. Considering Salmonella enterica subsp. enterica serovar Typhimurium and serovar Typhi as signature serovars, we classified the clades as CRISPR1-STM/cas-STM and CRISPR1STY/cas-STY, respectively. Serovars of the two clades displayed better relatedness, concerning CRISPR-1 leader and cas operon, across genera than between themselves. This signifies the acquisition of CRISPR1/Cas region a horizontal gene transfer event owing to the presence of mobile genetic elements flanking CRISPR1 array. The CRISPR2 tree does not show such relation. Spacer mapping of the two CRISPR arrays suggests the construct to be canonical, with only $8.8 \%$ spacer conservation among the serovars. As opposed to broad-host-range serovars, the host-specific serovars harbor fewer spacers. All typhoidal serovars have CRISPR1-STY/cas-STY system. Comparison of CRISPR and cas phenograms with that of multilocus sequence typing (MLST) suggests differential evolution of CRISPR/Cas system implying supplementary roles beyond immunity.
\end{abstract}

Key words: CRISPR; cas genes; Diversification; Evolution; MLST; Phylogenetic analysis

\title{
1.INTRODUCTION
}

Genus Salmonella is classified into two species, Salmonella enterica and Salmonella bongori. S. enterica further evolved into six subspecies (subsp.) enterica, salamae, arizonae, diarizonae, houtenae and indica ${ }^{1}$. Serovars of S. enterica subsp. enterica have diverse host range ranging from broad-hostrange, host-adapted to host-specific ${ }^{2}$ pertinent to within-host evolution ${ }^{3}$.

Before divergence, S. bongori and S. enterica acquired Salmonella pathogenicity island 1 (SPI-1 $)^{4}$ and later S. enterica laterally acquired SPI-2 (required for the survival in deep tissues and macrophages) thereby, enhancing its virulence potential ${ }^{4}$. As per the adopt-adapt model of bacterial speciation ${ }^{5}$, the adopted lateral gene(s) divert the evolution path promoting bacterial adaptation consequently increasing its fitness ${ }^{6}$. Over time, S. bongori and S. enterica horizontally acquired multiple virulence factors progressively enhancing their pathogenicity ${ }^{3}$.

The presence of the Clustered Regularly Interspaced Short Palindromic Repeats (CRISPR) and a cognate set of CRISPR-associated (cas) genes, has recently been related to the bacterial virulence potential $^{7}$. In E. coli, CRISPR units are negatively correlated with pathogenic potential where, the reduction in CRISPR activity is proposed to promote lateral gene transfer favoring its evolution ${ }^{8}$. Conversely, some reports demonstrate a positive correlation between the CRISPR and pathogenicity 
owing to virulence genes regulation ${ }^{9}$. S. enterica possesses type I-E CRISPR system with two CRISPR arrays, CRISPR 1 and CRISPR $2^{10}$, separated by $\sim 16 \mathrm{~kb}^{11}$. Many proto-spacers of this were traced on the chromosome instead of its general target - phages and plasmids ${ }^{12}$. This suggests a potential role of the CRISPR-Cas system in endogenous (virulence) gene regulation possibly regulating the pathogenesis of Salmonella ${ }^{13}$. To test the association of CRISPR-Cas system and pathogenicity, we studied the evolutionary dynamics of CRISPR-Cas system across 47 strains of Salmonella species. The evolution was studied in the light of three essential attributes of CRISPR-Cas components- the leader, the array, and the cas operon. We created a graphic map of the spacers of 131 strains across 22 serovars belonging to two species of Salmonella, comprehending the structural composition and its configuration. Next, the strains were assorted into two groups with respect to the CRISPR1 leader, spacer composition, and cas gene composition and similarity. This divergence was analyzed in comparison to multi-locus sequence typing (MLST) based on the seven core genes. Our study elucidates the hidden aspects and develops a strong correlation between the CRISPR-Cas system and the host specificity of various serovars of Salmonella, demonstrating that this system could have potential involvement in the virulence and pathogenicity.

\section{RESULTS}

\subsection{Diversity of the CRISPR arrays in Salmonella.}

We extracted all possible CRISPR1 and 2 arrays in correct orientation for 131 strains (Table S1, supplementary methodology). S. bongori and S. enterica subsp. enterica contained both CRISPR arrays while subsp. arizonae and subsp. diarizonae, had only one CRISPR array. One strain (str. NCTC10047), out of the six strains examined of subspecies arizonae, and all the eight strains of subspecies diarizonae had an intact CRISPR array.

We mapped the spacer sequences (Fig. S1) of all 131 strains illustrating the blueprint of spacer conservation among the strains within and across serovars. The acquisition of spacers is in a precise fashion with conserved configuration for a particular serovar while the differences lie in the presence or absence of spacer(s). The spacers of serovars Enteritidis, Heidelberg, and Typhi are highly conserved among all its strains, whereas the serovars Typhimurium, Newport, Anatum, Montevideo and Tennessee had significant variability in the spacer composition of both the CRISPR arrays (Fig. 1 and Fig. S1a). Among all strains, we identified 437 and 327 unique spacers in CRISPR1 and CRISPR2 arrays, respectively. The average abundance of spacers for CRISPR1 and CRISPR2 is 15.3 and 12.6, respectively (Table S2). CRISPR1 array of serovar Tennessee str. ATCC 10722 (63 spacers) and CRISPR2 array of serovar Typhimurium str. USDA-ARS-USMARC-1880 (35 spacers) are the largest (Fig. S1). CRISRP1 array of serovar Anatum, Dublin, Gallinarum, Pullorum and, Gallinarum/Pullorum (two spacers), and CRISPR2 array of serovars Sendai and Typhi (one spacer) are the shortest (Fig. S1). We observed duplication and triplication of spacer in some serovars (Fig. S1a-S1b). 
Strikingly, the analysis of the CRISPR arrays in serovars Montevideo and Saintpaul separated the respective strains into two groups with two distinct sets of unique and conserved spacers (Table S1). For serovar Montevideo, the two groups comprised eight and nine strains. However, CRISPR arrays of all the analyzed strains of serovar Saintpaul except strain SARA26 (an outlier) had similar spacer composition. These results suggest polyphyletic origin of serovars Montevideo and Saintpaul, similar to that reported for serovar Newport ${ }^{14}$. Notably, the broad-host-range serovars have multiple spacers, while the host-specific serovars have very few spacers (Fig. 1).

A bird's eye view on direct repeat (DR) sequence of the CRISPR arrays confirmed the sequence to be 29 bp long, partially palindromic ${ }^{12}$ and the last DR is degenerate ${ }^{15}$ (Fig. S2b, S2d \& S2f). The DR sequence is conserved within respective array across all the serovars except for the presence of few SNPs (Fig. S2a, S2c \& S2e). The degeneration of last DR is of varying degrees, primarily witnessed near the $3^{\prime}$ end, while the $5^{\prime}$ end substantially conserved. Considerable difference in the last DR of both the arrays (Fig. S2) suggests existence of heterogeneous termination mechanism of pre-crRNA processing

\subsection{Phylogeny and classification of the CRISPR arrays.}

\subsubsection{Evolutionary studies of the CRISPR loci.}

To understand the evolutionary pattern of Salmonella serovars concerning the CRISPR loci, we generated phylogenetic trees inclusive of the array and leader sequence (Fig. 2) for 47 shortlisted set of strains (Table S1). These robust phylograms signify predominant divergence of the CRISPR loci. The topology has been observed in most of the clades and sub-clades, as evidenced by their high level of confidence from either the bootstrap values or the aLRT (approximate likelihood ratio test) scores.

The CRISPR1 tree had two distinct clades with serovars Typhimurium and Typhi as signature serovars (Fig.2). Thus, we classified the corresponding CRISPR loci as CRISPR1-STM and CRISPR1-STY, respectively. The strains of serovars Saintpaul and Montevideo harboring these loci were defined as Saintpaul-STM/ Montevideo-STM, and Saintpaul-STY/ Montevideo-STY, respectively.

In CRISPR 2 phenogram (Fig. 2), S. bongori emerged as an outgroup for the entire tree, and serovar Paratyphi C seems to have evolved distinctly from other serovars of S. enterica subsp. enterica. The CRISPR2 tree topology and sub-lineages were very distinct from that of the CRISPR1 tree with serovars forming polyphyletic clades. For example, serovar Saintpaul-STY grouped with serovars Typhimurium, Newport III, Heidelberg; Sendai, Paratyphi A grouped with Montevideo-STM; and Newport II with Anatum. The clustering was distinct from that obtained in the CRISPR1 tree. This can be authenticated by the aLRT scores evaluating that each branch under review provides a significant likelihood gain (Fig. S3 \& S4) suggesting different evolutionary relationships of both the CRISPR loci. 


\subsubsection{Comparison of the spacer sequences in the light of CRISPR phylogeny.}

To check if the difference in the CRISPR1 and CRISPR2 trees is due to the differential spacer conservation, the inter-serovar spacer content was analyzed (query cover $>95 \%$ ). Only $\sim 8-9.5 \%$ of unique spacers (36/437: CRISPR1 and 31/327: CRISPR2) were shared by two or more serovars (Fig. S1C). Some serovars in CRISPR1-STM clade have common spacers justifying their clustering. For example, serovars Typhimurium, Heidelberg, Newport III, and Paratyphi C; and serovars Anatum and Saintpaul-STM share their anchor spacer. Entire spacer set of serovars Gallinarum, Pullorum, and Gallinarum/Pullorum are present in serovar Enteritidis (Fig. S1c). Similarly, few serovars of CRISPR1STY clade share some spacers, e.g., serovars Paratyphi A and Sendai; and serovars Bovismorbificans and Newport II; share spacers, but these serovars do not cluster as expected.

For the CRISPR2 tree, atypically, the first spacer of serovars Enteritidis, Gallinarum, Pullorum, and Gallinarum/Pullorum; and serovar Paratyphi A and serovar Sendai is conserved (Fig. S1d). Serovars Paratyphi A, Saintpaul-STY, Montevideo-STY, and Newport II have one spacer in common, which is the last spacer in all the serovars except Newport II. Serovars Typhi, Enteritidis, Paratyphi C, Gallinarum, Pullorum and, Gallinarum/Pullorum share their anchor spacer. However, the serovars with identical anchor spacer belong to different clusters.

In the CRISPR2 locus tree and CRISPR1-STY clade, the serovars are not always clubbed as per the spacer similarity.

\subsubsection{Categorization of the leader sequence in the light of CRISPR phylogeny.}

Multiple sequence alignment of respective CRISPR sequences across the serovars revealed a significant match in the DR and the leader sequence. The CRISPR1 DR is conserved across the strains of CRISPR1-STM, and CRISPR1-STY clades with an SNP in a few strains of serovar Anatum (Fig. S2) thereby indicating major influence of leader on the topology of the CRISPR1 tree. To confirm this, we constructed phylogenetic trees for CRISPR1 and CRISPR2 leaders (Fig. 3). The topology of the CRISPR2 leader tree more or less coincided with the CRISPR2 tree (Fig. 2 and 3; supplementary figure S4 and S7). The clustering pattern of serovars in the CRISPR1 tree and CRISPR1 leader tree is similar except higher substitutions and enhanced branching in CRISPR1 tree (Fig. 2 and 3; supplementary figure S3 and S6). This indicates that the major force for segregation of CRISPR1-STM and CRISPR1STY clades is the variation in the leader sequence, and the formation of sub-clades/sub-clusters is a result of shared spacers among the serovars.

The serovars of S. enterica subsp. enterica have two distinct types of CRISPR1 leaders (Fig. 3 and Fig. S5), justifying their divergence in two clades. One of the leader sequence is identical to that of Newport $\mathrm{II}^{11}$ and is also present in all the serovars of CRISPR1-STY clade. Serovars Enteritidis and Gallinarum cluster in the CRISPR1 leader tree (Fig. 3) with 100\% leader identity. Serovars Gallinarum/Pullorum and Pullorum separate from this cluster possessing an SNP (A to G transition) in the leader. On similar grounds, other serovars cluster or separate from their clustering partners. The CRISPR1 leader of $S$. 
bongori, and S. enterica subsp. arizonae and subsp. diarizonae maximally matched with that of CRISPR1-STM (Fig. 3 and Fig. S5) and hence grouped in the CRISPR1-STM clade.

The CRISPR2 leader sequence is highly conserved (with a few SNPs) among all the serovars of $S$. enterica subsp. enterica (Fig. 3 and Fig. S5). The variations due to SNPs explains the serovar clustering in the CRISPR2 leader tree. For instance, the leaders of serovars Paratyphi A and Typhi have 94\% sequence similarity hence segregated into separate clades while the serovars Paratyphi A and Sendai clubbed together with $100 \%$ similarity.

The analyses indicate the overall topology and clustering of strains in the CRISPR1 and CRISPR2 trees is a result of differences in the leader sequence, and the spacer conservation governs the sub-clustering of the strains. The aLRT parametric assessment too coincides with the Bayesian approach (Fig. S6 \& S7).

\subsubsection{MLST phenogram and its association with the CRISPR array.}

MLST is considered as a robust and widely accepted phylogenetic reconstruction of species ${ }^{16}$. Hence we generated a reference MLST tree for the shortlisted strains (Table S1 using concatenated allelic data of seven housekeeping genes (Fig. 4). The MLST tree topology reveals S. bongori to be a clear outgroup, and the most ancestral Salmonella lineage, consistent with the reported data ${ }^{17}$. All other serovars formed lineages within a serovar-specific cluster depicting to have evolved together as an individual taxon except serovar Saintpaul and Newport. In this light serovar Saintpaul turns out to be polyphyletic like serovar Newport ${ }^{18}$. Serovar Saintpaul str. SARA26 separated from all serovars of subspecies enterica while the serovar Saintpaul str. CFSAN004173 clustered with Typhimurium/Heidelberg/Newport II clade. Serovar Paratyphi A is closer to serovar Typhimurium with 98.8\% similarity in the seven genes than to serovar Typhi (98.6\% similarity). The CRISPR and MLST phenograms are discordant signifying independent evolution. However, serovars Montevideo-STM and Montevideo-STY possess the same housekeeping genes but differ in CRISPR arrays segregating in two groups in CRISPR1 and CRISPR2 phenograms. This analysis indicates an interlinked evolution of the CRISPR1 and the CRISPR2 arrays.

\subsection{Phylogeny and classification of the cas operon.}

\subsubsection{Diversification of cas operon and its association with the CRISPR1 array.}

Two distinct cas gene arrangements were obtained for the strains comprising CRISPR1-STY and CRISPR1-STM clade. The cas operon of the respective categories were denoted as cas-STY and casSTM. For cas-STY, the cas 3 gene is present as a complement and is singled out from the other cas genes by a gap of 357 nucleotides (561 for serovar Montevideo-STY) (Fig. S8). For cas-STM, the cas genes are contiguous but the cas 3 gene of serovar Montevideo-STM and S. enterica subsp. arizonae is degenerate having a premature stop codon. Moreover, we noticed structural heterogeneity within the 
cas-STM operon across CRISPR1-STM strains, with respect to its position to both the CRISPR loci and the cas gene composition (Fig. S8). The cas operon of S. bongori, S. enterica subsp. enterica, subsp. arizonae and subsp. diarizonae were termed as cas-STM.B, cas-STM.E, cas-STM.A, and cas-STM.D respectively.

\subsubsection{Evolutionary studies and conservation of cas operon in Salmonella.}

The cas operon's heterogeneity was further assessed through phylogenetic analysis of the cas 3 gene and the entire cas operon (Fig. 5 and supplementary fig. S10). Two clades and the clustering of serovars obtained in both the phenograms is far more analogous with the CRISPR1 phenogram. To gain insights into the serovar clustering in cas genes, we performed a detailed comparative analysis of cas operon. The analysis of all cas genes considered in concatenation revealed the highest nucleotide similarity (99\%) between subspecies arizonae and diarizonae and lowest (28.6\%) between the cas-STM and casSTY group (Fig. S9). Between cas-STM and cas-STY group, cas 1 shares the highest similarity (74.4 $78.8 \%$ nucleotide and 82.5 - $87 \%$ amino acid match) while cse 2 shares the lowest similarity (no significant nucleotide match and 35\% amino acid identity) (Fig. 5). The Cas3 nuclease differed substantially between the cas-STM and cas-STY categories (10.47 - 18.4\% nucleotide and 37.4 - 45\% amino acid match). However, the functionally important domains- HD domain, helicase $\mathrm{C}$ terminal domain, and the DEAD-box were nearly $75 \%$ similar. The csel gene, was quite distinct between the cas-STM and cas-STY categories. The functionally important residues of Cse1 (E. coli) include Gly (157), glycine-loop residues (159-161), Lys (268), Asn (353), Glu (354) and Ala (355) required for the recognition of PAM sequences ${ }^{19}$ and lysine residues (289-290) for recruiting Cas3 protein ${ }^{19}$. Most of these residues are conserved across cas-STM and cas-STY categories (Fig. S11). This indicates that even though the Cse1 and Cas 3 differs significantly between these serovars, their functionality remains conserved.

A noteworthy observation was made for the CRISPR1 and cas trees. In general, the serovars of the CRISPR1-STY/cas-STY clade are more evolved than those of the CRISPR1-STM/cas-STM clade (Fig. 2 and 5). Additionally, there is the latest evolution of the strains of Montevideo-STY compared to the other serovars. In contrast, the serovars of CRISPR1-STM/cas-STM clade, show limited evolution as interpreted from their scaled branching pattern. However, CRISPR2 phylogeny depicts a mixed mode of such evolution.

\subsection{Inter-genus analysis of the CRISPR-Cas system.}

The evolutionary history of the CRISPR and cas loci across the Enterobacteriaceae family was studied through comparative sequence analysis and phylogenetics. Only the CRISPR1 leader of Salmonella showed substantial match across genera Escherichia, Citrobacter, Shigella, and Klebsiella, while the CRISPR2 leader showed match only with Klebsiella (75\%). Thus, we constructed a CRISPR1 leader phenogram with 17 strains belonging to these genera (Table S3), and some strains of CRISPR1-STM 
and CRISPR1-STY clades. The phylogenetic tree diverged into two main clades (Fig. 6) similar to the CRISPR1 tree of Salmonella with the same signature serovars. The strains of CRISPR1-STY category grouped with Escherichia, Shigella and some strains of Citrobacter (Fig. 6 and Table S3) while the strains of CRISPR1-STM clustered with Klebsiella, and a strain of Citrobacter (Fig. 6).

The cas-STM operon showed $\sim 75 \%$ similarity with that of the species, Klebsiella pneumoniae (str. TGH10), Citrobacter freundii (sp. CFNIH3), and Shigella boydii (str. ATCC 49812), which is significantly higher than that with cas-STY (28.6\%). On the contrary, the cas-STY operon displayed $\sim 84 \%$ similarity with Citrobacter freundii (sp. CFNIH9) and Citrobacter (sp. 30_2). Intriguingly, the cas-STY showed only a $12 \%$ match with $E$. coli.

\subsection{CRISPR-Cas system flanked by mobile genetic elements (MGE).}

To decipher the probable involvement of horizontal gene transfer (HGT), we screened the presence of the signature genes, helicase, transposase, and integrase ${ }^{20,21}$ in the proximity of the CRISPR-Cas region of Salmonella and analyzed the GC content of this region in comparison to the whole genome. We found that out of 20 serovars, 18 serovars (representative strains of each considered) showed truncated/probable transposase, $30 \mathrm{~kb}$ upstream of the CRISPR1 loci (Fig. 7 and Table S4). Two partial but different amino acid sequences of transposase were obtained for serovars belonging to the CRISPR1-STM and CRISPR1-STY clades. The GC content of the CRISPR arrays for most of the serovars was higher than the GC content of the whole genome except a few serovars with smaller arrays had lower GC content due to AT rich leader sequence (Fig. 7 and Table S4). A transposase gene was also present upstream of CRISPR2 array in serovars Paratyphi A and Typhi. Helicase gene was present in serovars Typhi and Typhimurium.

\section{DISCUSSION}

The evolutionary mechanisms in bacteria are highly complex with environmental factors intricately modulating the genome architecture and functionality. This provides extra-ordinary resilience to the bacteria. When combined with HGT and recombination events the evolutionary framework of the bacteria is significantly influenced providing multiple opportunities for successful evolution as a potent pathogen. Our study depicts the evolution of Salmonella virulence with respect to CRISPR-Cas system that is involved in phage-immunity, pathogenicity, and genome evolution. We categorized this system into two types CRISPR1-STM/cas-STM and CRISPR1-STY/cas-STY based on the phylogenetic segregation and differences in the CRISPR1 leader and cas genes features of the shortlisted 47 strains. Similar segregation pattern was observed with a large set to 128 strains (Fig. S12). The CRISPR1-STM clade included strains that are host-adapted, host-specific, and have broad-host range (Fig. 2) ${ }^{22,23}$. The CRISPR1-STY clade comprised typhoidal serovars (Fig. 2) ${ }^{24}$, and serovars Montevideo, Newport II, 
Tennessee, Bovismorbificans and Saintpaul having broad host-range $e^{25,26}$ and association with outbreaks of human salmonellosis ${ }^{27-29}$.

The chronicles of battles between the bacteria and the invading MGE are registered as spacers in the CRISPR arrays. The divergence of the CRISPR-Cas system in Salmonella was understood through a comprehensive intra- and inter-serovar mapping of the spacer sequences. The conservation of spacers was weak across the serovars with only $\sim 8-9.5 \%$ of unique spacers present in more than one serovar. This suggests a non-random distribution of the arrays (to be precise, spacers) foreshadowing the evolutionary kinetics of Salmonella. The hyper-diversity of the spacers, but their conservation within a particular serovar confers acquisition of the spacers a very primitive event with different selective pressures operating on different serovars. This could have led to a non-classic evolutionary model of the CRISPR-Cas system. This polymorphism of spacers across serotypes finds utility in serotyping ${ }^{30,31}$.

The CRISPR-Cas evolution is complex and the modular character hinders its forthright categorization based on the serovar host-range and geographical location. Serovars Newport II and Newport III, both infect primates, reptiles and aves but still segregate into two separate clades in the CRISPR1 tree. Serovar Typhimurium strain SARA13 and serovar Saintpaul SARA26 were isolated from the same geographic location, France (GenBank database), but segregated into CRISPR1-STM and CRISPR1STY clades, respectively. Spacer conservation within strains of all the serovars irrespective of the geographic location suggests the roots of CRISPR origin to be ancient and the displacement has a diminutive role. Some serovars share a significant proportion of their spacers, suggesting their recent divergence in the evolutionary timeline of Salmonella. Example include, 66\% of CRISPR1 and 100\% of CRISPR2 spacers of serovar Heidelberg are identical with those of serovar Typhimurium.

The anchor spacer gives an indirect correlation of the last common ancestor (LCA) for the inter-serovar studies $^{11}$. Most serovars of the three individual clusters in CRISPR1-STM clade of CRISPR1 tree (Fig. 2), share the anchor spacer suggesting a LCA for each. For instance, in the Enteritidis cluster serovar Enteritidis and Pullorum share anchor spacer. Serovar Gallinarum shares spacers with Enteritidis but not the anchor spacer indicating the loss of some common spacers (also by other serovars) including the anchor spacer. In the CRISPR1-STY clade, some serovars do have similar spacers, but none is the anchor spacer. For the CRISPR2 tree, even though serovars Enteritidis, Paratyphi C, Gallinarum, Pullorum, Gallinarum/Pullorum, and Typhi are part of different clusters, they have an identical anchor spacer. This suggests that the LCA for these serovars could have been communal and diverged lately due to distinct evolutionary forces. Serovar Bovismorbificans share five CRISPR1 spacers with serovar Saintpaul-STM and anchor spacer with serovar Newport II indicating divergence from Newport II and recombination with Saintpaul-STM (from CRISPR1-STM clade). Generally, the anchor spacer for a particular serovar is conserved and disparities tolerated at the leader proximal end ${ }^{11}$. Intriguingly, all the strains of a given serovar have a few spacers conserved, irrespective of their position in the array and the spacer variability within the serovar. For instance, in CRISPR1, spacer B in the serovar 
Typhimurium, and spacers A \& P-R of serovar Newport III are highly conserved even though, these serovars show variations in their spacer composition (Fig. S1). One elucidation is, as implicated elsewhere ${ }^{13}$, the spacer composition of the system could potentially leverage the pathogenic potential possibly through endogenous gene regulation and, therefore, were retained in the system.

The cas genes of the strains in the cas-STM and cas-STY category are highly similar within each category but differ from the other, except for the cas 1 and cas 2 , genes required for spacer acquisition. However, the key residues of Cse1 and the functional domains of Cas3 are conserved indicating the conservation of their functionality. The strains comprising cas-STM and cas-STY are homologous to that of the CRISPR1 phenogram. This is empirical as the CRISPR1 array, and the cas operon lie in close proximity, suggesting an evolutionary coherence between the two. Furthermore, the strains belonging to CRISPR1-STY/cas-STY category showed higher substitutions per sequence site, implying the plasticity for new alterations.

The size of the spacer set for a given serovar is directly proportional to its host-range. Ubiquitous serovars like Typhimurium, Newport II, Tennessee, and Heidelberg has huge spacer set while hostspecific/adapted serovars like Typhi, Sendai, Gallinarum, Dublin etc. possess a few spacers. Considering the role of spacers in regulating endogenous genes ${ }^{32}$, we hypothesize that versatility in spacers opens up opportunities to infect multiple hosts permitting the regulation of a variety of genes. All the spacers of the host-specific serovars Gallinarum, Pullorum, and Gallinarum/Pullorum are present in serovar Enteritidis (a broad-host-range serovar) along with some additional spacers further testifying our hypothesis. Among the host-specific/adapted serovars, the primate-specific serovars, Typhi, Paratyphi A, and Sendai, have a CRISPR1-STY leader and cas-STY operon. The remaining four serovars are specific to poultry or cattle containing the CRISPR1-STM/cas-STM type CRISPR/Cas system. With these observations, we are tempted to put forward a conjecture for Salmonella serovars'Fewer spacers in CRISPR array restrict the host-range, and the CRISPR1-STY/cas-STY type CRISPR/Cas system confers specificity to primate'. However, in-depth analyses and further research are warranted to understand any advantage of having a CRISPR1-STY/cas-STY system in infecting primates.

The incongruence in CRISPR and cas trees with the MLST tree implies a possible event of HGT. We tracked the presence of a truncated transposase, a hallmark of HGT, $\sim 30 \mathrm{~kb}$ upstream of the CRISPR1 array with the protein sequences showing significant match within the serovars of the CRISPR1-STM (>95\%) and CRISPR1-STY (88\%) clades. A high GC content of the CRISPR array fortifies the occurrence of HGT event. A further support is evidenced through the histone-like nucleoid-structuring protein (H-NS) mediated regulation of cas operon in S. enterica subsp. enterica serovar Typhi ${ }^{33}$. H-NS is associated with HGT, acting as a transcriptional silencer of horizontally acquired genes by binding to the AT rich DNA and blocking RNA polymerase ${ }^{3}$. One may possibly argue the regulation of CRISPR array by H-NS through its AT-rich leader. With this background, we anticipate that H-NS could have originally silenced the CRISPR-Cas system and later evolved to regulate the functioning of cas operon 
and probably the CRISPR arrays. It is quite likely that all the strains belonging to CRISPR1-STY clade conform to this mechanism. While validation of such mechanism in the strains of CRISPR1-STM clade needs accreditation.

The CRISPR and cas attributes of S. bongori, S. enterica subsp. arizonae and subsp. diarizonae, justify their placement in 'CRISPR1-STM' and 'cas-STM' clades of CRISPR1 and the cas phenograms, thereby reflecting a higher similarity with CRISPR1-STM/cas-STM ( 85\%) than with CRISPR1STY/cas-STY (20-30\%). Surprisingly, the CRISPR1-STM/cas-STM and CRISPR1-STY/cas-STY sequences showed better relatedness with other genera of Enterobacteriaceae family namely, Escherichia, Klebsiella, Shigella, and Citrobacter than with each other. A significant proportion of the Escherichia, Shigella, and Klebsiella strains have the CRISPR/Cas system that matched with CRISPR1STM/cas-STM (unpublished data). Nevertheless, few strains of the Enterobacteriaceae family (Klebsiella \& Citrobacter) contain both the type of CRISPR1 array and cas operon. Moreover, in Citrobacter freundii complex sp. CFNIH3, a truncated transposase was found $30 \mathrm{~kb}$ upstream of the CRISPR1 loci with a $40 \%$ similarity in the connecting-region of S. enterica subsp. enterica serovar Typhimurium indicating occurrence of HGT event. The split of Salmonella serovars into two separate clades and clubbing of serovar of CRISPR1-STM with Shigella and E. coli was also observed in the Cas2 protein phylogram reported by Touchan et al. ${ }^{12}$ thus, validating our results.

With the comprehensive analysis of all the results, we put forward the following hypotheses for evolution of CRISPR-Cas system. Given that a significant proportion of Escherichia, Shigella, and Klebsiella strains harbor CRISPR1-STM/cas-STM type leader and operon, we propose that the LCA of the Enterobacteriaceae family could have had the CRISPR1-STM/cas-STM type loci. Further, after the divergence from Escherichia, Salmonella could have acquired its CRISPR2 array, as there exists no similarity in their leader sequences, while leaders of S. enterica and S. bongori are 78\% identical. S. enterica subsp. arizonae and subsp. diarizonae do not have a CRISPR2 array, which could have been possibly lost in due course of evolution. Many strains of subsp. arizonae do not contain the CRISPR1 array suggesting its loss as well. We also observed the conservation of CRISPR2 leader throughout $S$. enterica subsp. enterica, the existence of similar spacers of CRISPR2 array across the strains, and no bifurcation in the CRISPR2 phenograms. With this background, we propose the following. Apparently one, few or all the serovars belonging to the CRISPR1-STY/cas-STY clade could have acquired CRISPR1-STY leader and cas-STY operon from an unknown source, possibly by HGT event in the gut of primates, subsequently transmitting amongst other Salmonella strains and other genera whereas the CRISPR2 array remained unaffected. The inheritance of the CRISPR1-STY/cas-STY system perhaps renders competitive advantage to the strains possessing it, in terms of its pathogenicity and enhanced survival in hostile conditions. Further investigation of CRISPR-Cas evolution across Enterobacteriaceae family is warranted to strengthen our hypothesis. 
The results of the study hold prospect in providing insights into the evolution of Salmonella (other enteropathogens) as a pathogen with diverse host-specificity, linking various regulatory networks with the CRISPR-Cas system.

\section{MATERIALS AND METHODS}

\subsection{Sequence Data Collection.}

The CRISPR and cas loci of 131 Salmonella strains were obtained in correct orientation after retrieving the data from GenBank and CRISPR-Cas++ database ${ }^{34}$. For details, refer to supplementary material. For MLST, sequences of seven housekeeping genes namely, purE, hemD, aroC, dnaN, hisD, thrA and sucA were retrieved from BIGSdb software ${ }^{35}$, and the unannotated ones were extracted from the genome's annotation files using customized written bash script. The composite sequence tags were allocated for the allelic profiles of these seven genes.

The CRISPR leader sequences of 17 strains comprising of genus Salmonella, Escherichia, Citrobacter, Shigella, and Klebsiella were extracted using the CRISPR-Cas++ database/CRISPRCasFinder and matched with the Salmonella's CRISPR leader sequences.

\subsection{Analysis of the CRISPR-Cas components.}

To create spacer maps of the CRISPR arrays, the spacers were aligned to maximize their homology across the Salmonella strains. The intra- and inter-serovar spacer conservation were estimated using python scripts. The orientation of the individual cas genes was traced and the sequence similarity calculated using a custom python script. The amino acid sequences of Cse1 and the essential domains of Cas3 protein (HD domain, helicase C terminal domain, and the DEAD-box) of Salmonella were extracted from the Uniprot database and aligned with the reported sequences of E. coli using the tool Clustal Omega.

The sequence $\log$ o for the CRISPR leader and DR sequences ${ }^{34}$ were generated using the tool WEBLOGO ver 2.8.2 $2^{43}$. The MGEs elements were manually checked $50 \mathrm{~kb}$ upstream and downstream of each CRISPR loci using the annotated GenBank files. Further, the GC content of the CRISPR-Cas components, and the whole genome was computed using python script.

\subsection{Phylogenetic analysis.}

Multiple sequence alignment were performed on the aforesaid sequences by MUSCLE version 3.6 with default parameters ${ }^{36}$ integrated into Molecular Evolutionary Genetics Analysis version 10 (MEGA X) ${ }^{37}$. All positions with alignment gaps and missing data were excluded (complete deletion option). The resulting alignments of respective groups of sequences were used to construct each phylogenetic tree using the Maximum Likelihood (ML) method $^{38}$ guided by the most suitable evolutionary model proposed by Bayesian approach ${ }^{39}$. The trees were given confidence with a bootstrap value of 1000 
iterations. The substitution models and the parameters used for the reconstructed trees were TamuraNei model with Gamma distribution for MLST; Tamura 3-parameter model for CRISPR1 leader and CRISPR2 Leader; Kimura 2-parameter model for CRISPR1 leader with array and CRISPR2 leader with array; and Kimura 2-parameter model along with gamma distribution for concatenated cas genes and cas3 gene. The Newick format of the trees was used for further visualization and analyses through MEGA X and $\mathrm{TTOL}^{40}$. All trees were drawn to scale, and the branch lengths were calculated as the number of substitutions per site.

For topology validation, the phylogenetic trees for Salmonella were also reconstructed using the program PHYML version $3.1^{41}$ with statistical tests for branch support. The statistical parametric analysis of Shimodaira-Hasegawa re-estimation of log-likelihood was adopted to get the consensus maximum likelihood tree. The general time reversible substitution models were kept uniform for all the trees generated. Curation of the multiply aligned sequences was done through GBlocks, having, a stringent selection of many contiguous non-conserved positions being disallowed ${ }^{42}$.

\section{References}

1 Lamas, A. et al. A comprehensive review of non-enterica subspecies of Salmonella enterica. Microbiol Res 206, 60-73, doi:10.1016/j.micres.2017.09.010 (2018).

2 Gao, X. et al. Evolution of host adaptation in the Salmonella typhoid toxin. Nat Microbiol 2, 1592-1599, doi:10.1038/s41564-017-0033-2 (2017).

3 Ilyas, B., Tsai, C. N. \& Coombes, B. K. Evolution of. Front Cell Infect Microbiol 7, 428, doi:10.3389/fcimb.2017.00428 (2017).

4 Gal-Mor, O. Persistent Infection and Long-Term Carriage of Typhoidal and Nontyphoidal Salmonellae. Clin Microbiol Rev 32, doi:10.1128/CMR.00088-18 (2019).

5 Sheppard, S. K., Guttman, D. S. \& Fitzgerald, J. R. Population genomics of bacterial host adaptation. Nat Rev Genet 19, 549-565, doi:10.1038/s41576-018-0032-z (2018).

6 Brooks, A. N., Turkarslan, S., Beer, K. D., Lo, F. Y. \& Baliga, N. S. Adaptation of cells to new environments. Wiley Interdiscip Rev Syst Biol Med 3, 544-561, doi:10.1002/wsbm.136 (2011).

7 Shmakov, S. A. et al. The CRISPR Spacer Space Is Dominated by Sequences from Species-Specific Mobilomes. mBio 8, doi:10.1128/mBio.01397-17 (2017).

8 Karginov, F. V. \& Hannon, G. J. The CRISPR system: small RNA-guided defense in bacteria and archaea. Mol Cell 37, 7-19, doi:10.1016/j.molcel.2009.12.033 (2010).

9 García-Gutiérrez, E., Almendros, C., Mojica, F. J., Guzmán, N. M. \& García-Martínez, J. CRISPR Content Correlates with the Pathogenic Potential of Escherichia coli. PLoS One 10, e0131935, doi:10.1371/journal.pone.0131935 (2015).

10 Karimi, Z., Ahmadi, A., Najafi, A. \& Ranjbar, R. Bacterial CRISPR Regions: General Features and their Potential for Epidemiological Molecular Typing Studies. Open Microbiol J 12, 59-70, doi:10.2174/1874285801812010059 (2018).

11 Shariat, N., Timme, R. E., Pettengill, J. B., Barrangou, R. \& Dudley, E. G. Characterization and evolution of Salmonella CRISPR-Cas systems. Microbiology 161, 374-386, doi:10.1099/mic.0.000005 (2015).

12 Touchon, M. \& Rocha, E. P. The small, slow and specialized CRISPR and antiCRISPR of Escherichia and Salmonella. PLOS One 5, e11126, doi:10.1371/journal.pone.0011126 (2010).

13 Cui, L. et al. CRISPR-. Pathogens 9, doi:10.3390/pathogens9010053 (2020). 
14 Zheng, J. et al. Whole-Genome Comparative Analysis of Salmonella enterica Serovar Newport Strains Reveals Lineage-Specific Divergence. Genome Biol Evol 9, 10471050, doi:10.1093/gbe/evx065 (2017).

15 Richter, C., Chang, J. T. \& Fineran, P. C. Function and regulation of clustered regularly interspaced short palindromic repeats (CRISPR) / CRISPR associated (Cas) systems. Viruses 4, 2291-2311, doi:10.3390/v4102291 (2012).

16 Pérez-Losada, M., Arenas, M. \& Castro-Nallar, E. Microbial sequence typing in the genomic era. Infect Genet Evol 63, 346-359, doi:10.1016/j.meegid.2017.09.022 (2018).

17 McQuiston, J. R. et al. Molecular phylogeny of the salmonellae: relationships among Salmonella species and subspecies determined from four housekeeping genes and evidence of lateral gene transfer events. J Bacteriol 190, 7060-7067, doi:10.1128/JB.01552-07 (2008).

18 Porwollik, S. et al. Characterization of Salmonella enterica subspecies I genovars by use of microarrays. J Bacteriol 186, 5883-5898, doi:10.1128/JB.186.17.58835898.2004 (2004).

19 Hayes, R. P. et al. Structural basis for promiscuous PAM recognition in type I-E Cascade from E. coli. Nature 530, 499-503, doi:10.1038/nature16995 (2016).

20 McDonald, N. D., Regmi, A., Morreale, D. P., Borowski, J. D. \& Boyd, E. F. CRISPRCas systems are present predominantly on mobile genetic elements in Vibrio species. BMC Genomics 20, 105, doi:10.1186/s12864-019-5439-1 (2019).

21 Deng, Y. et al. Horizontal gene transfer contributes to virulence and antibiotic resistance of Vibrio harveyi 345 based on complete genome sequence analysis. BMC Genomics 20, 761, doi:10.1186/s12864-019-6137-8 (2019).

22 Anderson, C. J. \& Kendall, M. M. Serovar Typhimurium Strategies for Host Adaptation. Front Microbiol 8, 1983, doi:10.3389/fmicb.2017.01983 (2017).

23 Schröter, M. et al. Pet snakes as a reservoir for Salmonella enterica subsp. diarizonae (Serogroup Illb): a prospective study. Appl Environ Microbiol 70, 613-615, doi:10.1128/aem.70.1.613-615.2004 (2004).

24 Hiyoshi, H., Tiffany, C. R., Bronner, D. N. \& Bäumler, A. J. Typhoidal Salmonella serovars: ecological opportunity and the evolution of a new pathovar. FEMS Microbiol Rev 42, 527-541, doi:10.1093/femsre/fuy024 (2018).

25 Andino, A. \& Hanning, I. Salmonella enterica: survival, colonization, and virulence differences among serovars. ScientificWorldJournal 2015, 520179, doi:10.1155/2015/520179 (2015).

26 Jones, T. F. et al. Salmonellosis outcomes differ substantially by serotype. J Infect Dis 198, 109-114, doi:10.1086/588823 (2008).

27 Sheth, A. N. et al. A national outbreak of Salmonella serotype Tennessee infections from contaminated peanut butter: a new food vehicle for salmonellosis in the United States. Clin Infect Dis 53, 356-362, doi:10.1093/cid/cir407 (2011).

28 Plumb, I. D. et al. Outbreak of Salmonella Newport Infections with Decreased Susceptibility to Azithromycin Linked to Beef Obtained in the United States and Soft Cheese Obtained in Mexico - United States, 2018-2019. MMWR Morb Mortal Wkly Rep 68, 713-717, doi:10.15585/mmwr.mm6833a1 (2019).

29 Brandwagt, D. et al. Outbreak of. Euro Surveill 23, doi:10.2807/15607917.ES.2018.23.1.17-00335 (2018).

30 Fabre, L. et al. CRISPR typing and subtyping for improved laboratory surveillance of Salmonella infections. PLoS One 7, e36995, doi:10.1371/journal.pone.0036995 (2012).

31 Thompson, C. P. et al. High-Resolution Identification of Multiple Salmonella Serovars in a Single Sample by Using CRISPR-SeroSeq. Appl Environ Microbiol 84, doi:10.1128/AEM.01859-18 (2018).

32 Wimmer, F. \& Beisel, C. L. CRISPR-Cas Systems and the Paradox of Self-Targeting Spacers. Front Microbiol 10, 3078, doi:10.3389/fmicb.2019.03078 (2019). 
33 Medina-Aparicio, L. et al. The CRISPR/Cas immune system is an operon regulated by LeuO, H-NS, and leucine-responsive regulatory protein in Salmonella enterica serovar Typhi. J Bacteriol 193, 2396-2407, doi:10.1128/JB.01480-10 (2011).

34 Couvin, D. et al. CRISPRCasFinder, an update of CRISRFinder, includes a portable version, enhanced performance and integrates search for Cas proteins. Nucleic Acids Res 46, W246-W251, doi:10.1093/nar/gky425 (2018).

35 Jolley, K. A., Bray, J. E. \& Maiden, M. C. J. Open-access bacterial population genomics: BIGSdb software, the PubMLST.org website and their applications. Wellcome Open Res 3, 124, doi:10.12688/wellcomeopenres.14826.1 (2018).

36 Edgar, R. C. MUSCLE: multiple sequence alignment with high accuracy and high throughput. Nucleic Acids Res 32, 1792-1797, doi:10.1093/nar/gkh340 (2004).

37 Kumar, S., Stecher, G., Li, M., Knyaz, C. \& Tamura, K. MEGA X: Molecular Evolutionary Genetics Analysis across Computing Platforms. Mol Biol Evol 35, 15471549, doi:10.1093/molbev/msy096 (2018).

38 Jin, G., Nakhleh, L., Snir, S. \& Tuller, T. Inferring phylogenetic networks by the maximum parsimony criterion: a case study. Mol Biol Evol 24, 324-337, doi:10.1093/molbev/msl163 (2007).

39 Tamura, K. Estimation of the number of nucleotide substitutions when there are strong transition-transversion and $\mathrm{G}+\mathrm{C}$-content biases. Mol Biol Evol 9, 678-687, doi:10.1093/oxfordjournals.molbev.a040752 (1992).

40 Letunic, I. \& Bork, P. Interactive Tree Of Life (iTOL) v4: recent updates and new developments. Nucleic Acids Res 47, W256-W259, doi:10.1093/nar/gkz239 (2019).

41 Guindon, S. \& Gascuel, O. A simple, fast, and accurate algorithm to estimate large phylogenies by maximum likelihood. Syst Biol 52, 696-704, doi:10.1080/10635150390235520 (2003).

42 Dereeper, A. et al. Phylogeny.fr: robust phylogenetic analysis for the non-specialist. Nucleic Acids Res 36, W465-469, doi:10.1093/nar/gkn180 (2008).

43 Crooks, G. E., Hon, G., Chandonia, J. M. \& Brenner, S. E. WebLogo: a sequence logo generator. Genome Res 14, 1188-1190, doi:10.1101/gr.849004 (2004).

\section{Acknowledgments}

This work was supported by the Department of Science and Technology, Science and Engineering Research Board (Grant No. ECR_2017_002053) to SAM. The authors acknowledge the support of the Department of Biological Sciences, Sunway University, Selangor, Malaysia for providing the computational facilities.

\section{Author contributions}

S.K.K initiated the research and completed the main work under the S.A.M and C.L. guidance. B.A. carried out the computational assessments. S.A.M finalized the paper. All authors reviewed the manuscript.

\section{Competing interests}

The authors declare they have no competing interests. 


\section{Figure Legends}

FIGURE 1. Graphic map of spacer conservation in CRISPR1 (a) and CRISPR2 (b) array for Salmonella serovars. The shades of grey represent the conservation percentage of a given spacer in all the strains of the respective serovar where, the darker box indicate the presence of spacer in most of the strains (black: 100\%) while, the lighter box indicate the presence of spacer in a few strains.

FIGURE 2. The phylogeny of CRISPR leader+array, CRISPR1 (a), and CRISPR2 (b) across Salmonella serovars. The entire CRISPR arrays and their leader sequences were aligned using Muscle, and the phylogenetic trees were constructed by ML. The trees were visualised by iTOL.

FIGURE 3. The phylogeny and conservation of CRISPR leaders. (a \& b) Phylogeny of leaders across Salmonella serovars for CRISPR1 (a), and CRISPR2 (b) leaders. The CRISPR leader sequences were aligned using Muscle, and the phylogenetic trees were constructed by ML. The trees were visualised by iTOL. (c) The matrix depicting the inter-species and inter-subspecies conservation of the leader sequence of both the CRISPR arrays. The values represent the percent nucleotide identity with respect to the entire query cover. The reference strains are S. enterica serovar Typhimurium str.14028S, Typhi str. CT18, S. enterica subsp. arizonae str. NCTC10047, S. enterica subsp. diarizonae str. MZ0080 and S. bongori str. SA19983605.

FIGURE 4. The MLST phylogeny. The phylogenetic tree was constructed using the concatenated sequence of seven housekeeping genes - purE, hemD, aroC, dnaN, hisD, thrA, and sucA. The sequences were aligned using Muscle, and the phylogenetic trees were constructed by ML. The trees were visualised by iTOL.

FIGURE 5. The phylogeny and conservation of cas genes. (a $\boldsymbol{\&} \mathbf{b})$ Phylogeny of cas genes across Salmonella serovars entire cas operon (a) and the cas3 gene (b). The sequences were aligned using Muscle, and the phylogenetic trees were constructed by ML. The trees were visualised by iTOL. (c) Conservation of all the individual cas gene and Cas protein sequences. The amino acid percent conservation is depicted in parenthesis. The term 'ND' represents no nucleotide sequence similarity based on the default parameter of the tool Nucleotide-BLAST. The reference strains are S. enterica serovar Typhimurium str.14028S, Typhi str. CT18, S. enterica subsp. arizonae str. NCTC10047, S. enterica subsp. diarizonae str. MZ0080 and S. bongori str. SA19983605.

FIGURE 6. The Phylogeny of the CRISPR1 leader sequence of 17 strains of species of Enterobacteriaceae family. The CRISPR leader sequences were aligned using Muscle, and the phylogenetic trees were constructed by ML. The bootstrap values are indicated at each node. KP - 
Klebsiella pneumoniae, C - Citrobacter, SE-A - S. enterica subsps. arizonae, SE-D - S. enterica subsps. diarizonae, STM - S. enterica subsp. enterica serovar Typhimurium, STY - S. enterica subsp. enterica serovar Typhi, SE-NP - S. enterica subsp. enterica serovar Newport, SB - S. bongori, SB1 Shigella boydii and EC - Escherichia coli.

FIGURE 7. Generalised representation of the signature genes involved in horizontal gene transfer. All Salmonella serovars except serovars Bovismorbificans and Gallinarum/Pullorum contain the transposase gene upstream of CRISPR1 loci. * - transposase upstream of CRISPR2 is present only in serovars Typhi and Typhimurium. 
bioRxiv preprint doi: https://doi.org/10.1101/2020.07.08.193417; this version posted July 8, 2020. The copyright holder for this preprint (which was not certified by peer review) is the author/funder, who has granted bioRxiv a license to display the preprint in perpetuity. It is made available under aCC-BY-ND 4.0 International license.

Figures

Figure 1

(a)

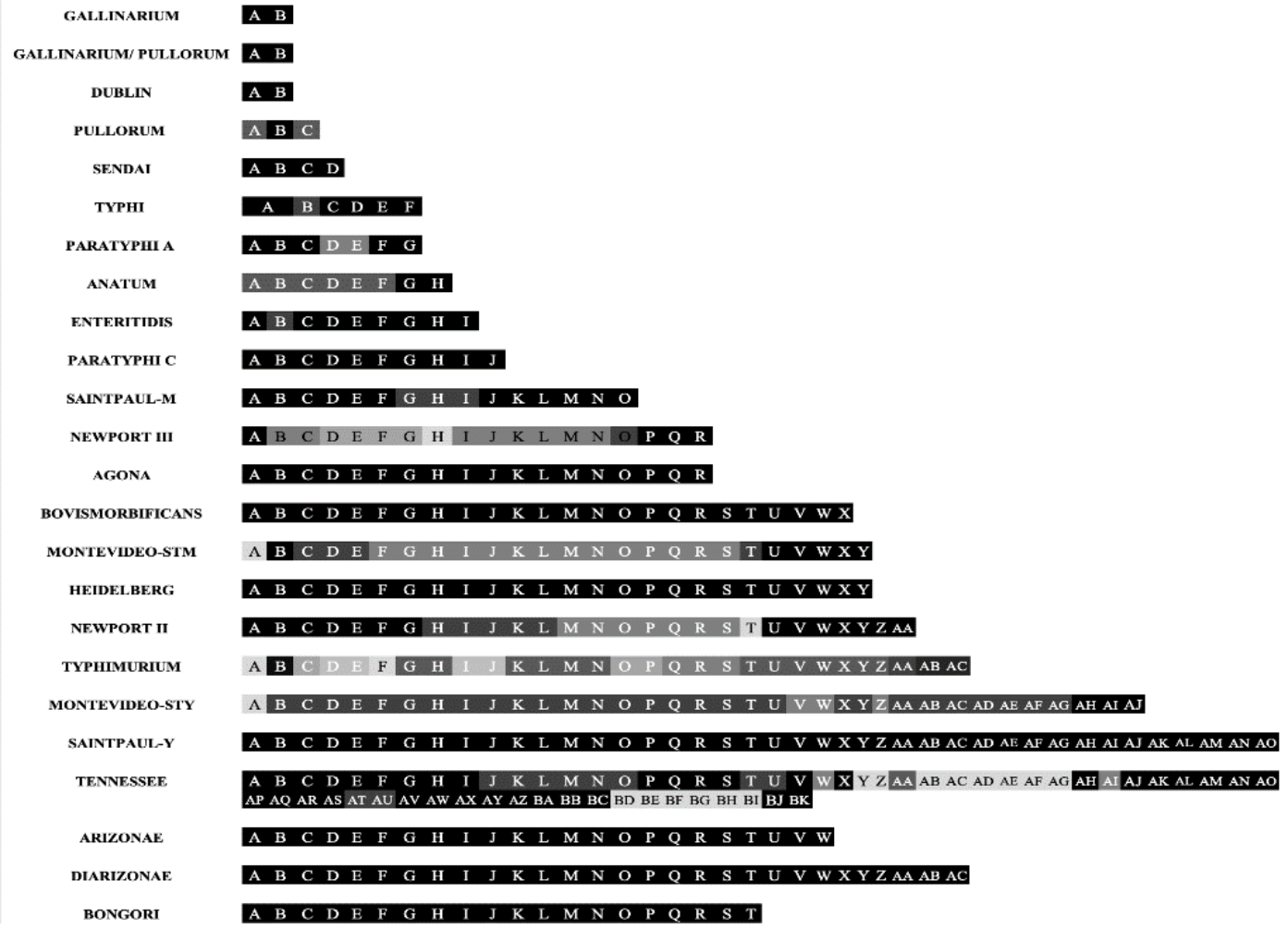

(b)
TYPHI
A
SENDAI A
PARATYPHI A A B C
DUBLIN A B C D E
PULLORUM A B C D E F
GaLLINARIUM/A B C D E F
AGONA ABCDEFGH
PARATYPHIC A B C DEFGH I
GaLlinarium A B C D E F G H I J
ENTERITIDIS A B C DEF G H I J K L
BOVISMORBIFICANS A B C D E F G H I J K L M N O
HEIDELBERG
$\triangle B C D E F G I J K L M N O P Q R$
NEWPORT II
A B C DEFGH I J K L M N QR S T U
NEWPORT III
$\triangle$ B C D E F G H I J K L M N OP QRS T U
SAINTPAUL-STM
A B C DEFGH I J MOPQR TUV
SAINTPAUL-STY
ABCDEFGHIJKLMNOPQRSTUV
TENNESSEE
A B C DE FG H I J K L M N OPQR S T U VW
MONTEVIDEO-STY
CDEF G H I J K L M NOPQR S T U VW
ANATUM
A B C D E F G H I JKL M N P Q R S TU VWXYZ
MONTEVIDEO-STM
A B C D EF G H I J K L M N O P R T U V XYZAA
TYPHIMURIUM

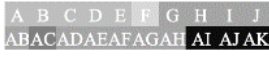
BONGORI
ABCDEFGHIJKLMNOPQ 
bioRxiv preprint doi: https://doi.org/10.1101/2020.07.08.193417; this version posted July 8, 2020. The copyright holder for this preprint (which was not certified by peer review) is the author/funder, who has granted bioRxiv a license to display the preprint in perpetuity. It is made available under aCC-BY-ND 4.0 International license.

Figure 2

(a)

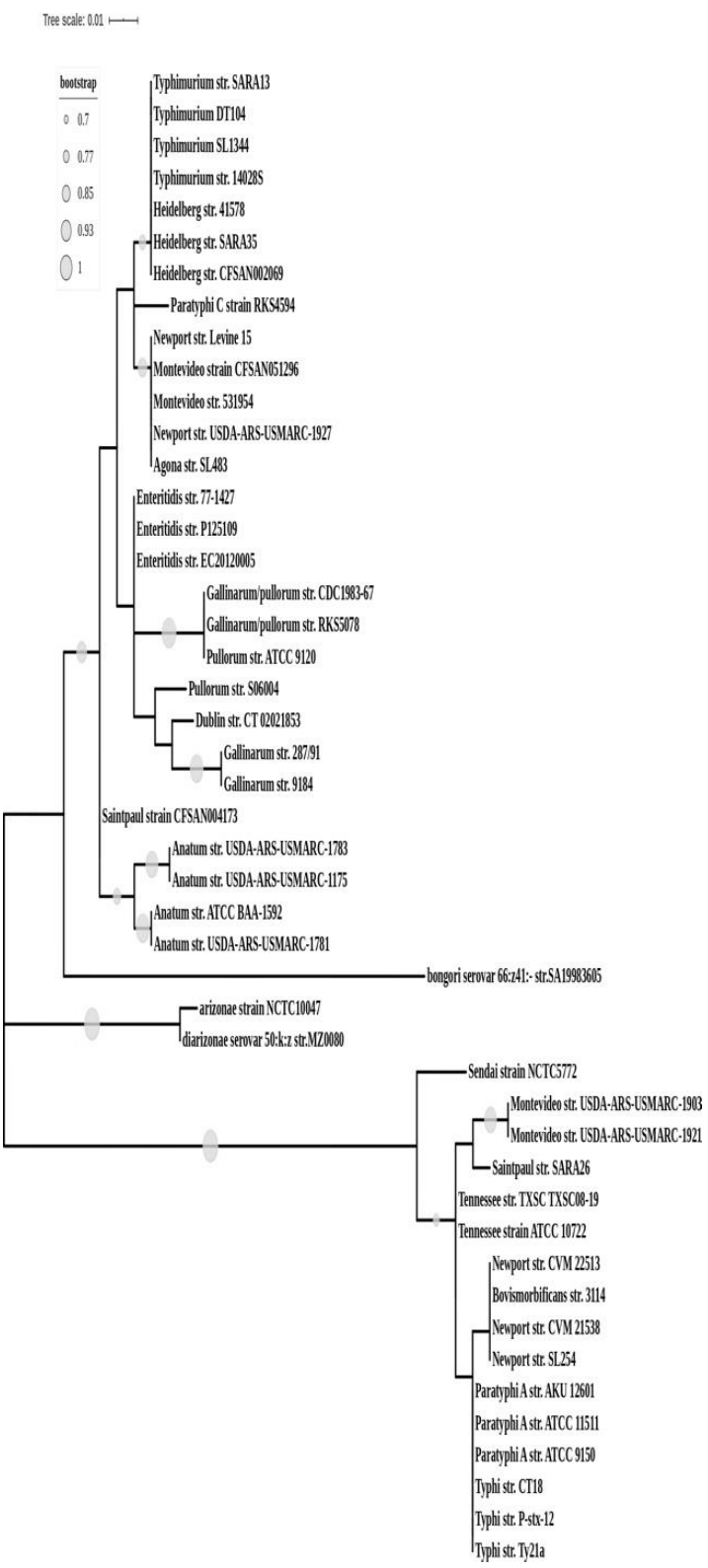

(b)

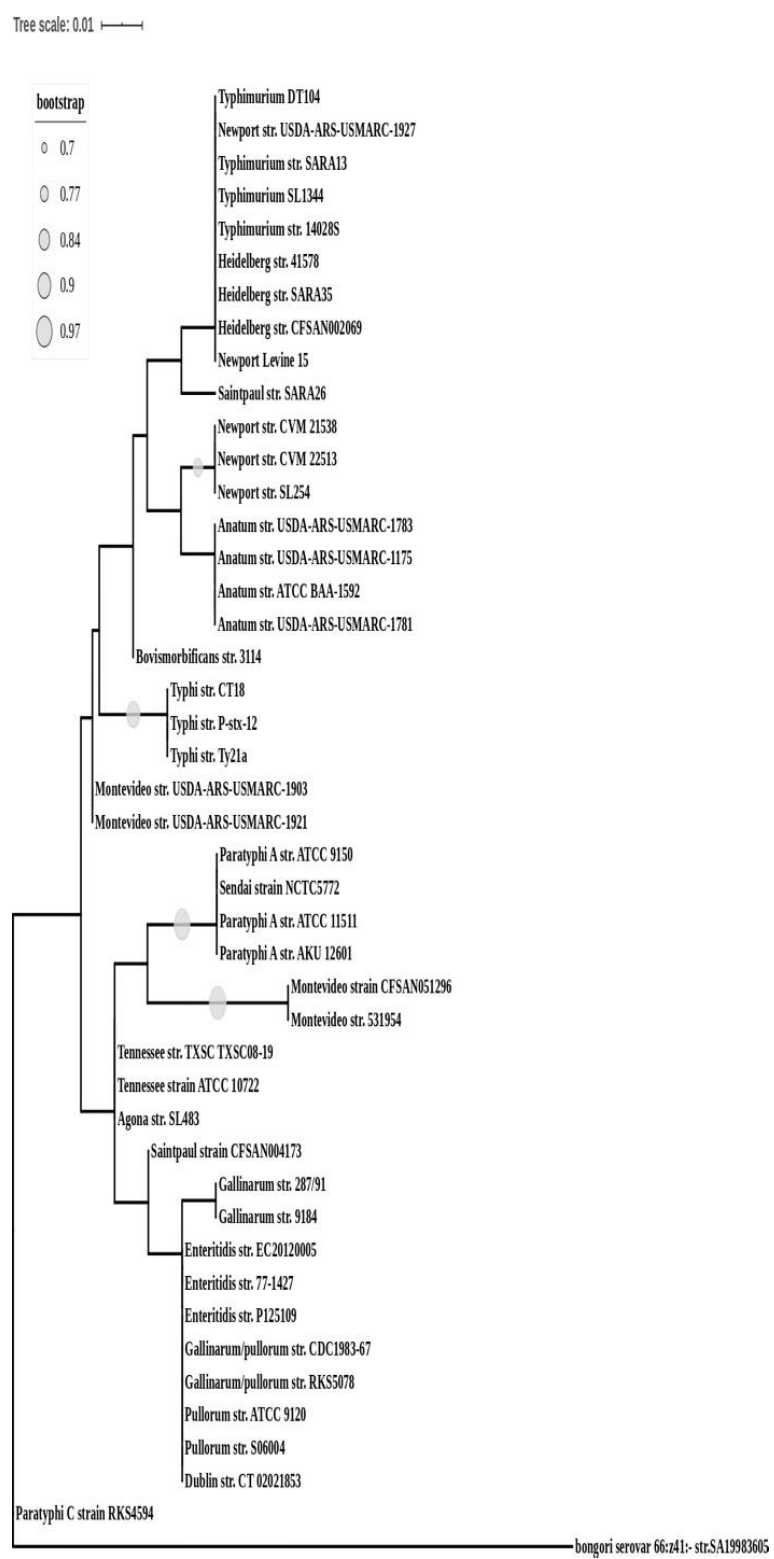


bioRxiv preprint doi: https://doi.org/10.1101/2020.07.08.193417; this version posted July 8, 2020. The copyright holder for this preprint (which was not certified by peer review) is the author/funder, who has granted bioRxiv a license to display the preprint in perpetuity. It is made available under aCC-BY-ND 4.0 International license.

Figure 3

(a)

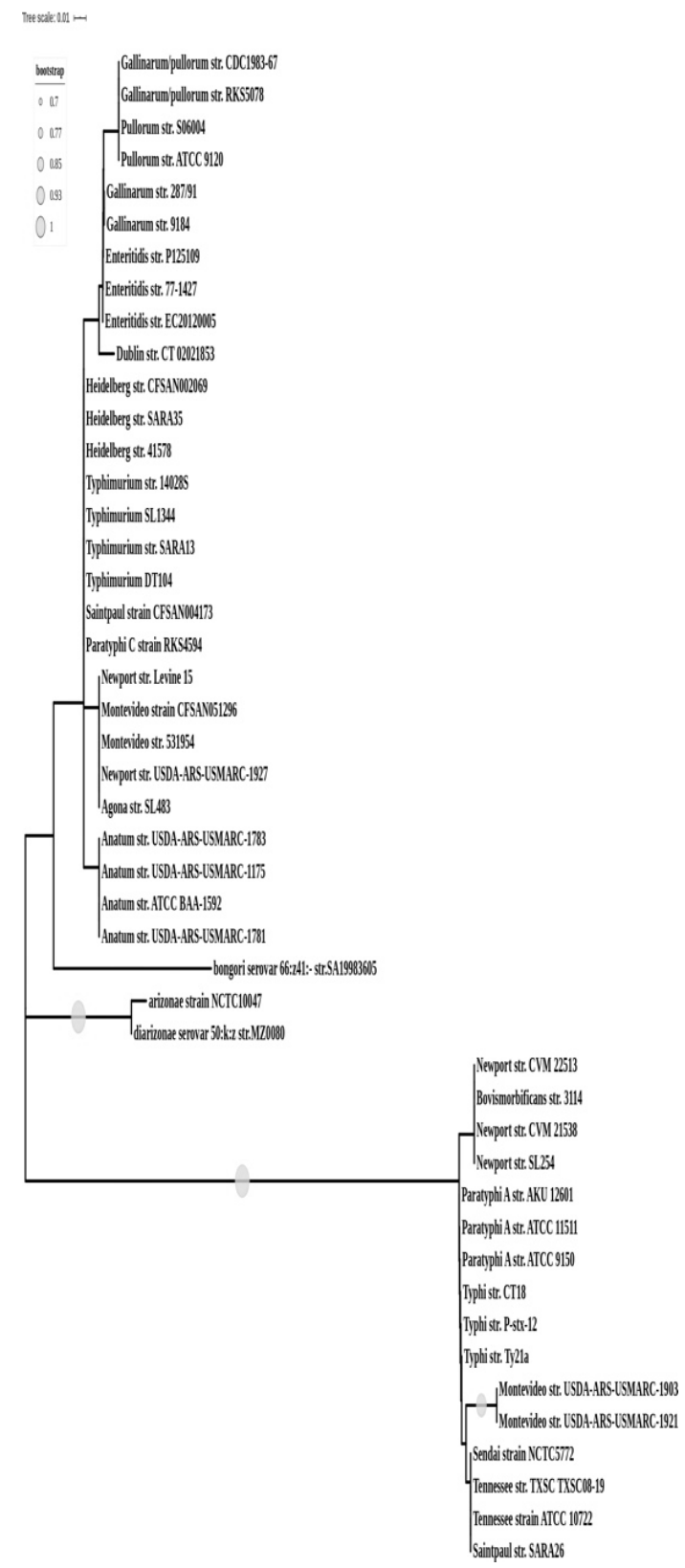

(b)

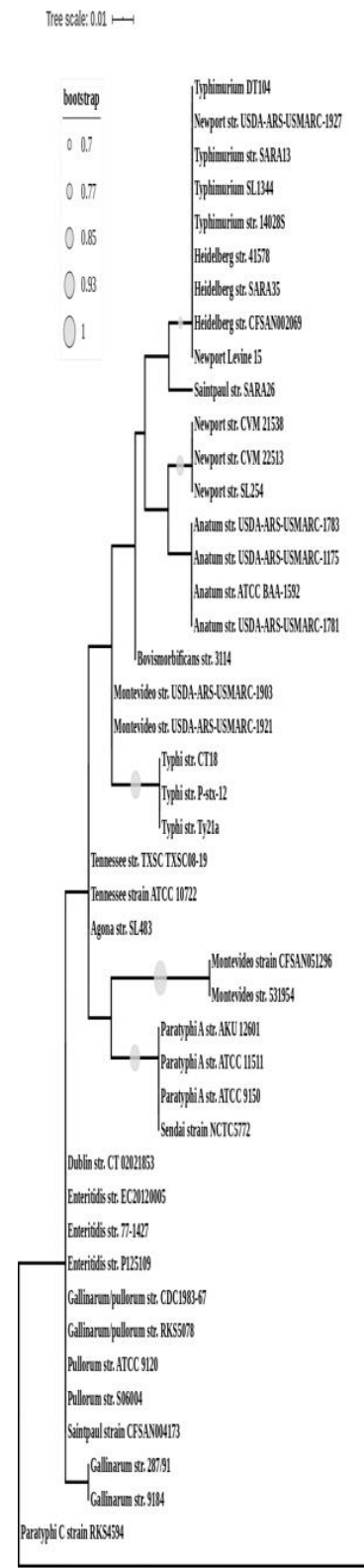

(c)

\begin{tabular}{|c|c|c|c|c|c|c|c|}
\hline & & \multicolumn{3}{|c|}{$\begin{array}{c}\text { Salmonella enterica subsp. } \\
\text { enterica }\end{array}$} & \multirow{2}{*}{\begin{tabular}{|c|}
$\begin{array}{c}\text { Salmonella } \\
\text { enterica } \\
\text { subsp. } \\
\text { arizonae \& } \\
\text { diarizonae }\end{array}$ \\
CRISPR 1 \\
\end{tabular}} & \multicolumn{2}{|c|}{ Salmonella bongori } \\
\hline & & $\begin{array}{c}\text { CRISPR } 1 \\
\text { STM }\end{array}$ & $\begin{array}{c}\text { CRISPR } 1 \\
\text { STY }\end{array}$ & CRISPR2 & & CRISPR 1 & CRISPR 2 \\
\hline \multirow{3}{*}{$\begin{array}{c}\text { Salmonella enterica } \\
\text { subsp. enterica }\end{array}$} & $\begin{array}{c}\text { CRISPR } \\
1 \text { STM }\end{array}$ & & $63.50 \%$ & $65.00 \%$ & $84.60 \%$ & $82.70 \%$ & $64.60 \%$ \\
\hline & $\begin{array}{c}\text { CRISPR } \\
1 \mathrm{STY}\end{array}$ & & & $55.40 \%$ & $59.60 \%$ & $65.40 \%$ & $62.20 \%$ \\
\hline & $\left|\begin{array}{c}\text { CRISPR } \\
2\end{array}\right|$ & & & & $72.10 \%$ & $64.10 \%$ & $78.60 \%$ \\
\hline \begin{tabular}{|c} 
Salmonella enterica \\
subsp. arizonae \& \\
diarizonae \\
\end{tabular} & $\left|\begin{array}{c}\text { CRISPR } \\
1\end{array}\right|$ & & & & & $75.00 \%$ & $68.00 \%$ \\
\hline \multirow{2}{*}{ Salmonella bongori } & $\begin{array}{c}\text { CRISPR } \\
1 \\
\end{array}$ & & & & & & $63.70 \%$ \\
\hline & $\begin{array}{c}\text { CRISPR } \\
2\end{array}$ & & & & & & \\
\hline
\end{tabular}


bioRxiv preprint doi: https://doi.org/10.1101/2020.07.08.193417; this version posted July 8, 2020. The copyright holder for this preprint (which was not certified by peer review) is the author/funder, who has granted bioRxiv a license to display the preprint in perpetuity. It is made available under aCC-BY-ND 4.0 International license.

Figure 4

Tree scale: $0.01 \mapsto$

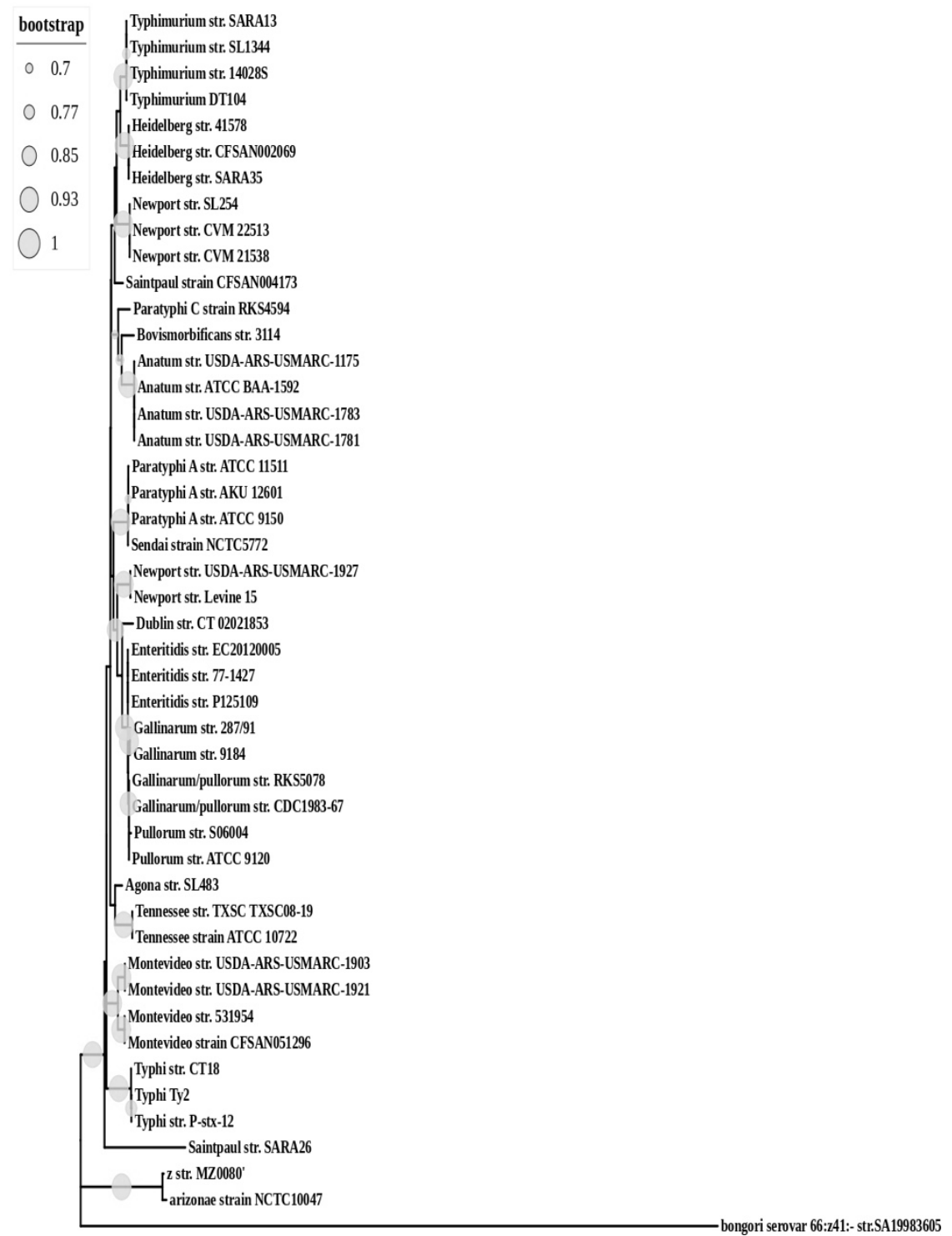


bioRxiv preprint doi: https://doi.org/10.1101/2020.07.08 193417; this version posted July 8, 2020. The copyright holder for this preprint (which was not certified by peer review) is the author/funder, who has granted bioRxiv a license to display the preprint in perpetuity. It is made available under aCC-BY-ND 4.0 International license.

Figure 5

(a)

0.7
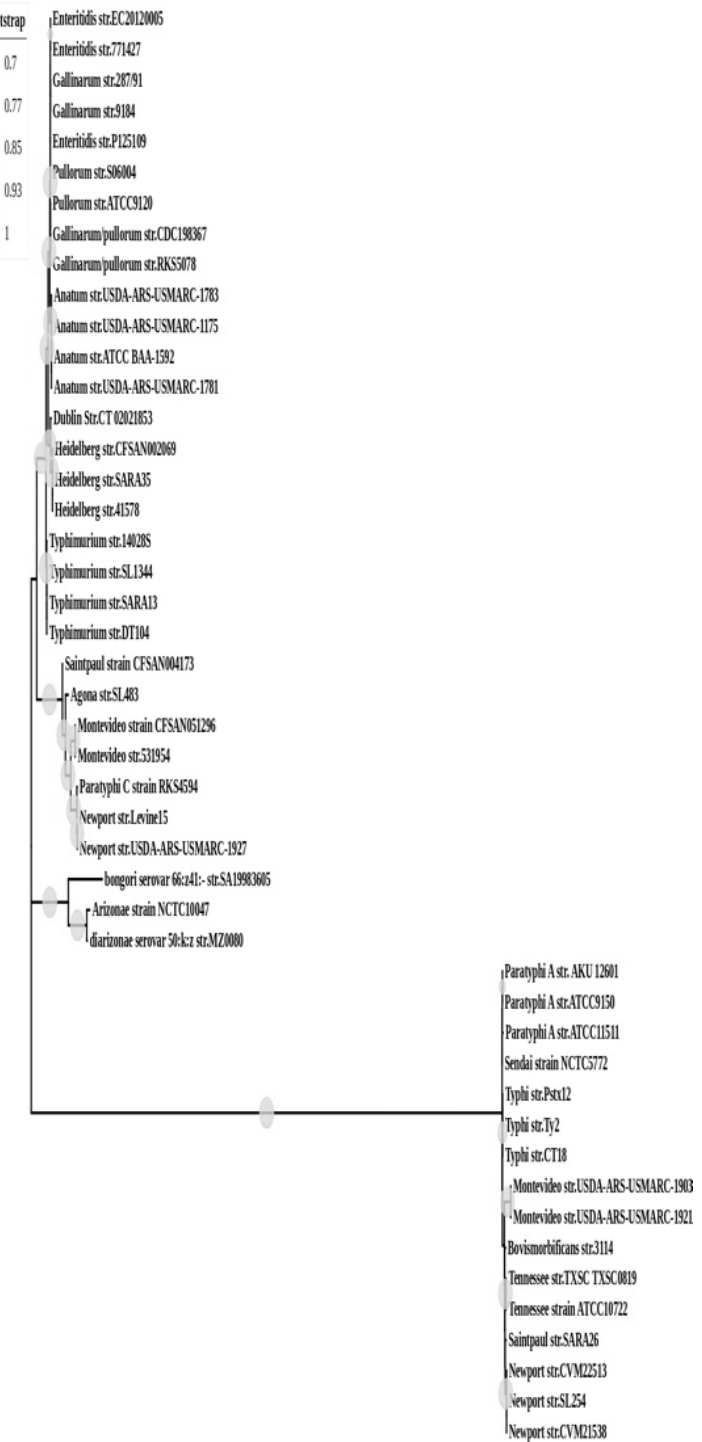

(b)

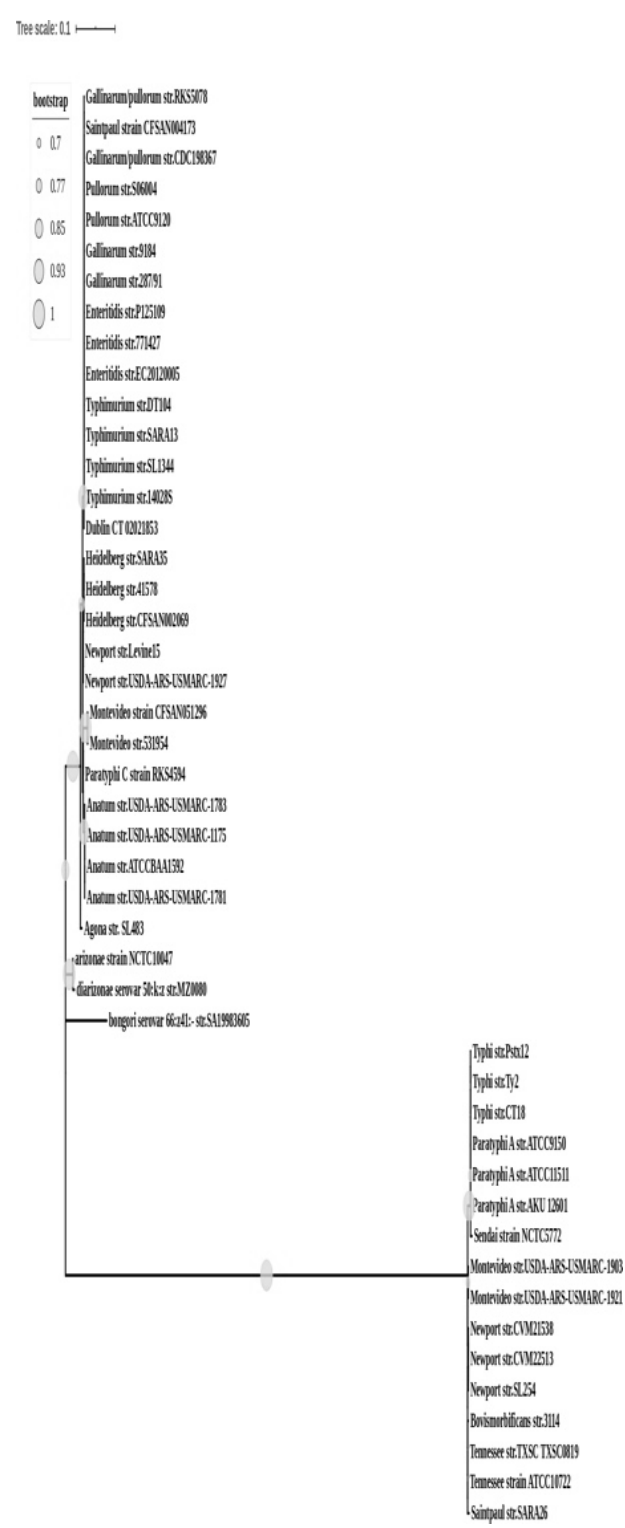

(c)

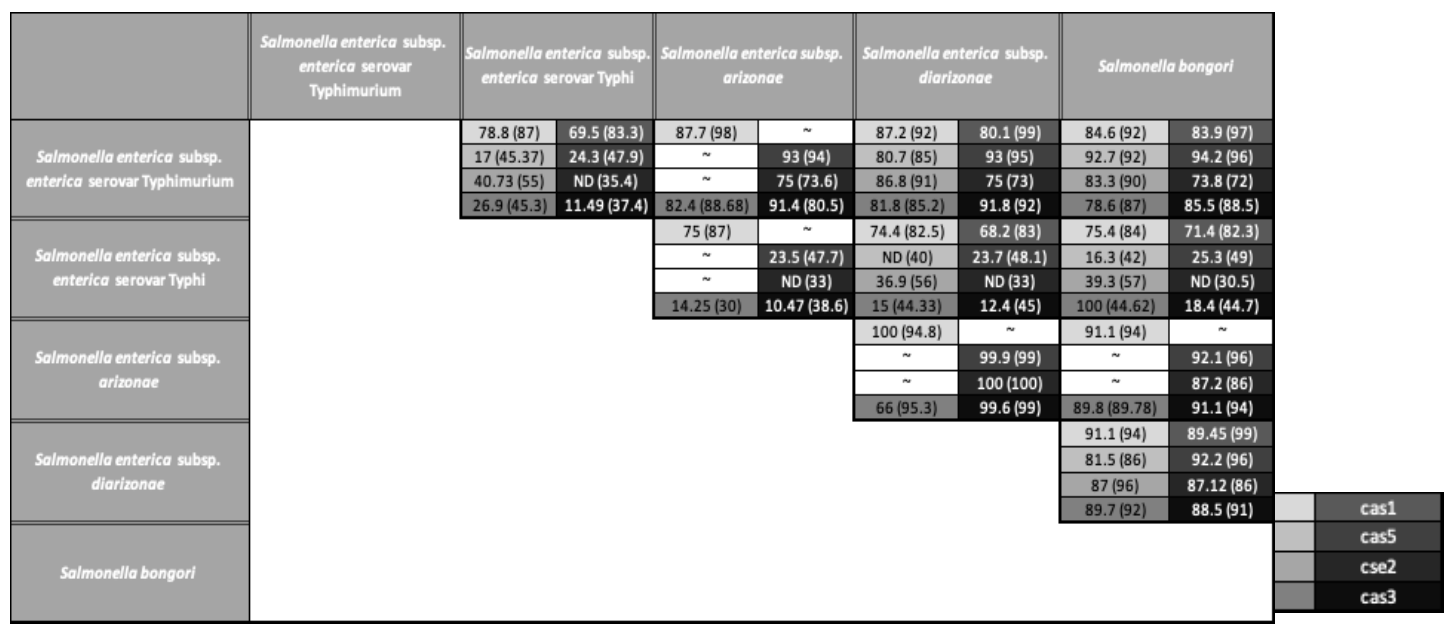


bioRxiv preprint doi: https://doi.org/10.1101/2020.07.08 193417; this version posted July 8, 2020. The copyright holder for this preprint (which was not certified by peer review) is the author/funder, who has granted bioRxiv a license to display the preprint in perpetuity. It is made available under aCC-BY-ND 4.0 International license.

Figure 6

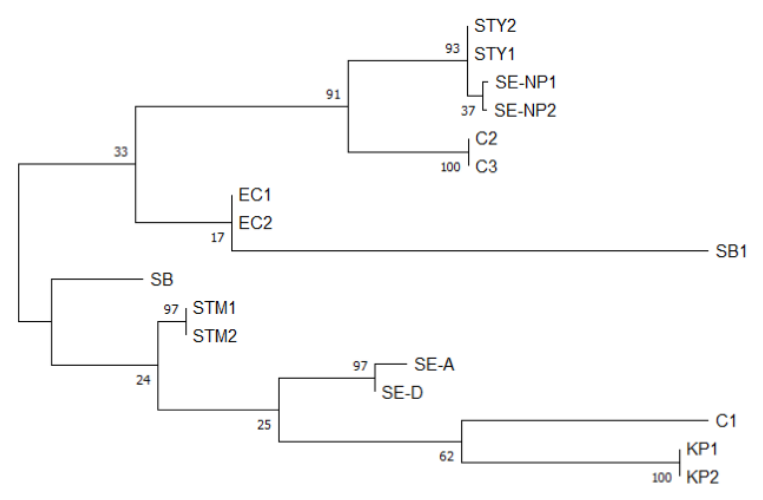

Figure 7

\section{Whole genome}

GC Content

$52 \%$

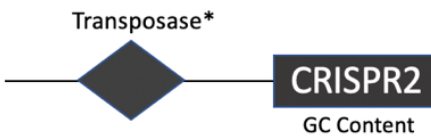

